\title{
CHEMICAL ABUNDANCES OF THE SECONDARY STARS IN THE BLACK HOLE BINARY A0620-00 AND THE NEUTRON STAR BINARY CEN X-4
}

\author{
J. I. González-Hernández, ${ }^{1}$ R. Rebolo, ${ }^{1,2}$ G. Israelian, ${ }^{1}$ and J. Casares ${ }^{1}$
}

We have determined abundances in the secondary stars of the black hole X-ray binary A0620-00 and the neutron star Binary Cen $\mathrm{X}-4$. These are $\mathrm{K}$ type stars veiled by the emission produced by the respective accretion discs. We searched for evidence of nucleosynthetic products from the progenitor of the compact object that could have contaminated the secondary stars (as in Israelian et al., 1999).

Using high resolution spectra obtained with VLT/UVES, we have derived in a consistent way stellar parameters and the veiling caused by the accretion disc. We have used a $\chi^{2}$ minimization procedure to explore a grid of 1500000 LTE synthetic spectra computed for a plausible range of both stellar and veiling parameters. Adopting the best model parameters obtained with this procedure, we have determined atmospheric abundances of $\mathrm{Fe}, \mathrm{Ca}, \mathrm{Ti}, \mathrm{Ni}$ and Al (see Table 1). In Figure 1 element abundances relative to iron are shown in comparison with the corresponding galactic trends in the relevant range of metallicities, taken from Feltzing \& Gustafsson (1998) and Bodaghee et al. (2003). We find the Li abundance in both systems is unusally high for this type of stars as it was previously noticed by Martin et al. (1994) suggesting that there is either a $\mathrm{Li}$ production mechanism of that the systems are young (less than $\sim 300 \mathrm{Myr}$ ).

In the secondary of $\mathrm{A} 0620-00$, the Fe abundance is slightly higher than solar. $[\mathrm{Ca} / \mathrm{Fe}]$ ratio of this star is consistent with abundances of metal-rich stars, while $\mathrm{Ni}$ and $\mathrm{Ti}$ appear to be moderately enhanced. $\mathrm{Al}$ is clearly over-abundant. Taking into account the small orbital separation, the mass transfer rate and the mass of the convection zone of the secondary star, a comparison with element yields in supernova explosion models (Umeda \& Nomoto, 2002, 2003) suggests a possible explosive event at the formation of the compact object.

In the secondary of Cen X-4, we do not find evidence of contamination of nucleosynthetic products from the progenitor of the neutron star. This suggests that the amount of matter captured by the

\footnotetext{
${ }^{1}$ Instituto de Astrofísica de Canarias, La Laguna, Código Postal E-38200, Tenerife, España. (jonay@ll.iac.es)

${ }^{2}$ Consejo Superior de Investigaciones Científicas, España.
}

TABLE 1

STELLAR ABUNDANCES

\begin{tabular}{lcc}
\hline & \multicolumn{2}{c}{$[\mathrm{E} / \mathrm{H}]_{L T E}$} \\
\hline Element & $\mathrm{A} 0620-00$ & Cen X-4 \\
\hline $\mathrm{Fe}$ & $0.14 \pm 0.20$ & $0.14 \pm 0.08$ \\
$\mathrm{Ca}$ & $0.10 \pm 0.20$ & $0.05 \pm 0.17$ \\
$\mathrm{Ti}$ & $0.37 \pm 0.23$ & $0.05 \pm 0.19$ \\
$\mathrm{Ni}$ & $0.27 \pm 0.10$ & $0.08 \pm 0.14$ \\
$\mathrm{Al}$ & $0.40 \pm 0.12$ & $0.05 \pm 0.12$ \\
\hline \multicolumn{2}{c}{$\log \epsilon(\mathrm{E})_{L T E}$} \\
\hline $\mathrm{Li}$ & $2.31 \pm 0.21$ & $2.56 \pm 0.21$ \\
\hline
\end{tabular}
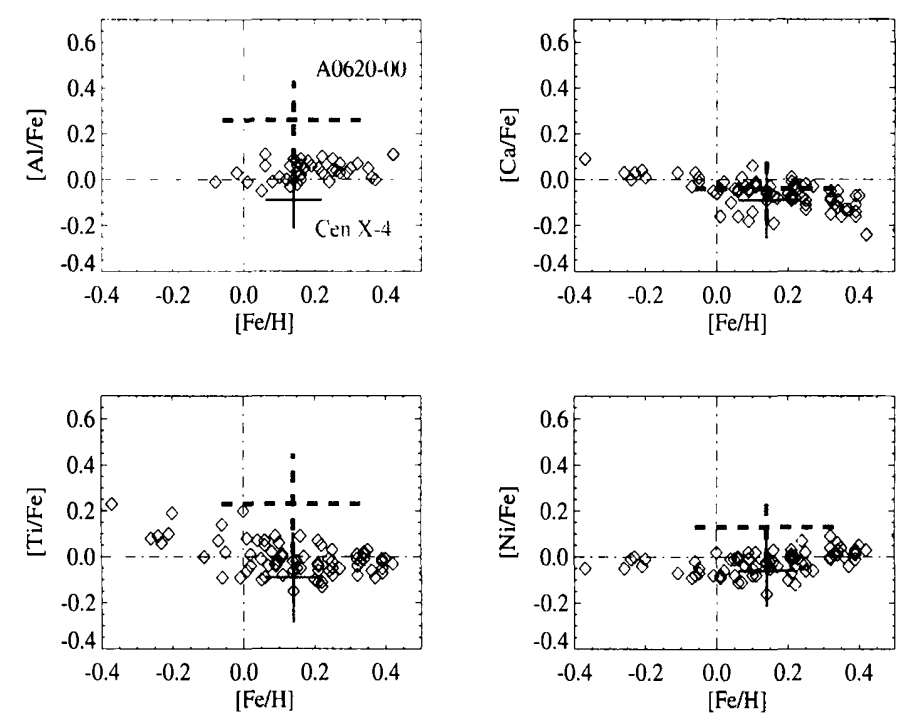

Fig. 1. Abundance ratios of the secondary stars in A0620-00 (thick dashed line cross) and Cen X-4 (thin solid line cross) in comparison with galactic trends

companion was negligible in comparison with the mass of its convection zone at the time of the explosion of the progenitor. This could have been the case if the neutron star had formed as a consequence of an asymmetric supernova explosion.

\section{REFERENCES}

Bodaghee, A., Santos, N. C., Israelian, G., Mayor, M., A\&A, 404: 715 (2003)

Feltzing, S., Gustafsson, B., Ap\&SS, 129: 237 (1998)

Israelian, G., Rebolo, R., Basri, G., Casares, J., Martín, E. L., Nature, 401: 142 (1999)

Martín, E. L., Rebolo, R., Casares, J., Charles, P. A., ApJ, 435: 791 (1994)

Umeda, H., Nomoto, K., ApJ, 565: 385 (2002)

Umeda, H., Nomoto, K., Nature, 422: 871 (2003) 